\title{
Efectos de la práctica de aikido sobre mindfulness y la ansiedad en estudiantes universitarios de Costa Rica \\ Effects of practicing aikido on mindfulness and anxiety in Costa Rican university students \\ *Rodrigo Cuéllar Hidalgo, *Aldo Bazán Ramírez, **Gerardo Alonso Araya Vargas \\ *Universidad Autónoma del Estado de Morelos (México), **Universidad de Costa Rica (Costa Rica)
}

\begin{abstract}
Resumen. El propósito del estudio fue examinar el efecto de la práctica del aikido sobre el mindfulness y el estado de ansiedad, en estudiantes universitarios sin experiencia previa en artes marciales. Se utilizó un diseño cuasi experimental intra sujetos con mediciones Pre y Post tratamiento, con un grupo control activo (estudiantes de Educación Física). Se midió mindfulness con la escala MAAS y la ansiedad con la escala de Hamilton. Se aplicó un entrenamiento centrado en el aprendizaje y práctica de diversas técnicas de aikido (waza) y de la forma en que debían ser recibidas dichas técnicas (ukemi), por 11 semanas (2 sesiones semanales de 2 horas cada una). Grupo experimental: n=12, con edades entre 18 y 62 años. Grupo control: $\mathrm{n}=12$ estudiantes, con edades entre 21 y los 34 años. Resultados: la práctica de aikido mostró tamaños de efecto significativos y de magnitud moderada tanto en mindfulness, como en la ansiedad. La edad no explica estos hallazgos. Se justifican estudios de seguimiento.
\end{abstract}

Palabras Claves: aikido, mindfulness, artes marciales, deporte, ansiedad, Psicología.

Abstract. The purpose of the study was to examine the effect of practicing aikido on mindfulness and anxiety state in university students with no previous experience in martial arts. We used an intra-subjects quasi-experimental design with Pre and Post treatment measurements, with an active control group (physical education students). Mindfulness was measured with the MAAS scale, whereas anxiety with the Hamilton scale. A training program focused on learning and practicing various aikido techniques (waza), and the way in which these techniques (ukemi) should be received, was implemented during 11 weeks (2 weekly sessions of 2 hours each). Experimental group: $n=12$, with ages between 18 and 62 years old. Control group: $\mathrm{n}=12$ students, with ages between 21 and 34 years old. Results: the practice of aikido showed significant effect sizes of moderate magnitude in both mindfulness and anxiety. Age does not explain these findings. Follow-up studies are recommended.

Key words: aikido, mindfulness, martial arts, sport, anxiety, psychology.

\section{Introducción}

En la literatura existen diversas acepciones en español para referirse al término mindfulness, entre las que se encuentran: «conciencia plena», «atención plena” y «presencia plena” (Baer, 2003; Davidson et al., 2003; Kabat-Zinn, 1982, 1990, 2003, 2005; Vallejo, 2006; Varela, Thompson, \& Rosch, 1992), y todas ellas hacen referencia a un estado que implica la atención del individuo centrada en la fenomenología de la experiencia, tanto de eventos internos como de eventos externos, que se registra momento a momento, siempre manteniendo una actitud de aceptación, evitando los juicios y las valoraciones (Kabat-Zinn, 2003).

Se ha explorado el mindfulness en diversos campos, como el educativo (De la Fuente, Franco, \& Mañas, 2010), el de la salud (Carlson \& Garland, 2005), el laboral (Shapiro, Astin, Bishop, \& Cordova, 2005), el campo social (Franco \& Navas, 2009) y el psicofisiológico (Lutz, Brühl, Scheerer, Jäncke, \& Herwig, 2016; Tang, Hölzel, \& Posner, 2015).Asimismo, se ha reportado beneficios de técnicas promotoras de mindfulness, para tratar la depresión (Baer, 2003; Cebolla \& Miró, 2007; Kumar, Feldman, \& Hayes, 2008; Segal, Williams, \& Teasdale, 2006), la ansiedad (Kabat-Zinn, 1990; Orsillo, Roemer, Block-Lerner, \& Tull, 2004; Roemer \& Orsillo, 2007; Shapiro, Carlson, Astin, \& Freedman, 2006; Vøllestad, 2016), el bienestar general y los síntomas de la percepción de dolor (Majumdar, Grossman, Dietz-Waschkowski, Kersig, \& Walach, 2002; Sharon et al., 2016; Williams, Kolar, Reger, \& Pearson, 2001; Zeidan et al., 2016; Zeidan et al., 2015; Zeidan \& Vago, 2016).

Esas técnicas de meditación, así como otros sistemas contemplativos (Delgado, 2009; Malmgren-Olsson,Armelius, \&Armelius, 2001; Marlatt \& Kristeller, 1999; Salmon, Lush, Jablonski, \& Sephton, 2009), clasificadas terapéuticamente como prácticas mente-cuerpo, pueden favorecer estados de conciencia como el mindfulness, pero este fenómeno también puede ocurrir, dada su naturaleza, ligado a prácticas deportivas o de ejercicio físico. Entre estas prácticas, se podría destacar las artes marciales, como el aikido, cuyas características sugieren una relación con el mindfulness.

El aikido es definido como una meditación en movimiento y se le atribuye incrementos en la consciencia de sus practicantes (Dobson y

Fecha recepción: 29-11-17. Fecha de aceptación: 08-05-18

Aldo Bazán Ramírez

abazan@uaem.mx
Miller, 1993; Saotome, 1986/2013, 1989). Esos cambios suelen explicarse por la naturaleza del entrenamiento en aikido, enfrentando situaciones marciales, con presión física y emocional constantes, ejecutando ataques y buscando neutralizar las acciones del rival, exigiendo para ello, que sus practicantes estudien durante el mismo entrenamiento, los sutiles cambios en el balance, esfuerzo y tiempo de cada movimiento, $y$ que sean conscientes de su propia respiración, equilibrio, centro de gravedad y de su conexión con su compañero de entrenamiento (Edinborough, 2011; Shioda, 1968, 1991; Ueshiba, 1988).

Recientemente se ha empezado a investigar la relación entre el aikido (y otras artes marciales y formas de ejercicio físico) y el mindfulness. Entre estos trabajos, Lothes, Hakan y Kassab (2013), publicaron en un mismo artículo dos estudios: uno no experimental y otro experimental. En su primer estudio Lothes et al. (2013) encontraron mayor mindfulness en principiantes o con bajo nivel de conocimiento de aikido (Kyu), en comparación con sujetos no entrenados en aikido ni otra arte marcial, y los sujetos con grados mayores de conocimiento (cinturones negros o Dan) fueron superiores en mindfulness que los otros dos grupos. Además, en su estudio experimental encontraron que los valores de mindfulness de un grupo que entrenó aikido (sin experiencia previa) y fue seguido por unos nueve meses, tendieron a mejorar en comparación con los de un grupo control conformado por estudiantes de un curso de Psicología. Según estos dos estudios, la práctica de aikido podría explicar cambios favorables en los niveles de mindfulness, pero sus resultados no son concluyentes dado su diseño estadístico.

También se ha investigado el efecto de la meditación para favorecer el mindfulness, en atletas (Röthlin, Birrer, Horvath, \& Grosse Holtforth, 2016), e incluso, se han desarrollado programas basados en mindfulness para población escolar(Volanen et al., 2016). Esto demuestra, la vigencia de esta línea de investigación.

Además, el aikido se ha relacionado con el perfil de estado anímico de sus practicantes (Pieter \& Pieter, 2008). Pero, falta investigar los efectos del aikido en estados anímicos como la ansiedad, variable que se ha evidenciado que es afectada positivamente por otras modalidades de ejercicio físico y deporte (Ensari, Greenlee, Motl \& Petruzzello, 2015; Fox, 1999; Petruzzello, Landers, Hatfield, Kubitz \& Salazar, 1991; Wegner et al., 2014; Wipfli, Rethorst \& Landers, 2008). Pero no está claro si esos efectos positivos sobre la ansiedad reportados en la literatura científica, se deben a aumentos en mindfulness o si podrían explicarse por otra causa. La ansiedad es importante para explicar el desempeño físico (González, Valdivia, Cachón, Zurita, \& Romero, 2017; Pérez-Ordás, Hernández-Hernández, \& García-Sánchez, 2011; 
Rodriguez-Pomeda, Casani, \& Sandoval, 2018), además de su importancia para la salud (Landers et al., 1991).

Al momento de realizar el presente estudio, no se han encontrado investigaciones que examinen experimentalmente la relación entre práctica de aikido, ansiedad y mindfulness. Por tanto, este aspecto también resulta de interés en el presente estudio, cuyo objetivo general fue examinar los efectos de la práctica de aikido sobre mindfulness y la ansiedad en estudiantes universitarios de Costa Rica.

\section{Método}

Esta investigación fue de tipo experimental, con mediciones pre y post tratamiento, con un grupo experimental y uno control que por las características de sus integrantes (sujetos físicamente activos, como se verá más adelante) se denomina como control activo. Los grupos no pudieron ser creados aleatoriamente, dada su condición de estudiantes matriculados en horarios preestablecidos por la administración de la universidad donde se llevó a cabo el trabajo (esta información se amplía más adelante). Por tanto, el diseño del estudio fue cuasiexperimental (Hernández, Fernández, \& Baptista, 2014; Thomas, Nelson, \& Silverman, 2015).

Como hipótesis se planteó que la práctica de aikido provoca aumentos en mindfulness y disminución en ansiedad en estudiantes universitarios sin experiencia previa en esta forma de ejercicio físico.

\section{Participantes}

Veinticuatro estudiantes de la Universidad de Costa Rica(UCR). El grupo experimental se formó con 12 estudiantes de diversas carreras (10 hombres y 2 mujeres; edades entre 18 y 62 años; 28.67士12.43); y el grupo control con 12 estudiantes del Bachillerato en Ciencias del Movimiento Humano (9 hombres y 3 mujeres; edades entre 21 y 34 años; 25 \pm 3.38 ).

\section{Instrumentos}

Mindfulness Attention Awareness Scale [MAAS] (Brown \& Ryan, 2003), versión en español traducida y validada en población costarricense por Araya-Vargas, Gapper-Morrow, Moncada-Jiménez y Buckworth (2009). La escala está conformada por 15 ítems que emplean una escala tipo Likert con valores desde 1='Casi siempre' hasta 6='Casi Nunca'. Puntajes altos indican mayor mindfulness. Tiene adecuada consistencia interna (alfa de Cronbach de versión original $=.80$ .87, y en español $=.88$ ) y validez de criterio (correlaciones estadísticamente significativas $\mathrm{r}=.31$ - .33, con la Mindfulness/Mindlessness Scale de Bodner y Langer, 2001) y constructo, mediante análisis factoriales confirmatorios reportados por Brown y Ryan (2003). El instrumento mide la capacidad del participante para estar atento, consciente y receptivo sobre lo que ocurre en el momento presente (Araya-Vargas et al., 2009; Brown \& Ryan, 2003). Posteriormente se han desarrollado otras versiones en español (Barajas \& Garra, 2014; López-Maya et al., 2015; Soler et al., 2012), que al igual que la publicada por Araya-Vargas et al. (2009), reportan adecuadas propiedades psicométricas.

Hamilton Anxiety Scale [HAS] (Hamilton, 1959), en su versión en español elaborada por Carrobles, Costa, Del Ser y Bartolomé (1987). Está conformada por 14 ítems que emplean una escala tipo Likert, cuyas posibles respuestas van del 0 ('Ausente') al 4 ('Muy Grave [Incapacitante]'). Tiene aceptable consistencia interna (alfa de Cronbach de .79 a .86) y validez concurrente con otras escalas de ansiedad como The Global Rating Anxiety de Covi ( $r=.73$ - .75) y el Inventario de Ansiedad de Beck ( $r=.56)$, entre otras propiedades psicométricas. Esta prueba puede ser aplicada por un evaluador post entrevista y también hay versiones que se pueden completar en línea (Asociación Catalana para el Tratamiento de la Ansiedad y Depresión, s.f.). Para el presente estudio se desarrolló (al igual que se hizo con la MAAS) una versión en línea. Con este instrumento se obtiene un componente emocional (psicológico) de la ansiedad, un componente somático y un puntaje global, al sumar los valores de respuesta a los ítems correspondientes (Carrobles et al., 1987).

\section{Procedimiento}

Para conseguir la muestra se ofertó un curso mediante la Unidad de Programas Deportivos, Recreativos y Artísticos (UPDRA-UCR) y de la Escuela de Educación Física y Deportes (EDUFI-UCR), titulado: Taller Recreativo de Aikido. Por cuestiones prácticas y de espacio disponible (el que fue asignado por la universidad), para el desarrollo de las sesiones, solo se ofertaron 25 plazas. Se inscribieron 24 estudiantes.

Para el grupo control, se solicitó a un grupo avanzado (estudiantes de tercer y cuarto nivel) del Bachillerato en Ciencias del Movimiento Humano de EDUFI-UCR, que respondieran los instrumentos.

Con ambas muestras definidas y tras una reunión donde se les solicitó leer y firmar, si estaban de acuerdo, el correspondiente consentimiento informado, se procedió a aplicar el pretest de las variables mencionadas. Para esto, se facilitó a todos los participantes un usuario y un password para entrar a un sitio web diseñado y creado específicamente para este estudio, para responder los instrumentos.

Luego, se procedió a la aplicación del programa en el grupo experimental a lo largo de 22 sesiones, realizando dos sesiones semanales de dos horas de duración, centradas en el aprendizaje y práctica de una selección de diversas técnicas de aikido (waza) así como de la forma en que debían ser recibidas dichas técnicas (ukemi).

Cada sesión mantuvo como estructura típica, un calentamiento previo de 20 minutos, un repaso de 40 minutos de las técnicas vistas la clase anterior y una hora para practicar las técnicas correspondientes al día.

Para diseñar el programa de entrenamiento, se seleccionaron técnicas del catálogo del estilo HEIAN RYU, creado por el Sensei Bruno Orozco León en 2004 en México (patente en trámite). Esas técnicas pueden dividirse en dos categorías: técnicas de control e inmovilización y técnicas de proyección. Se enseñaron cuatro técnicas de cada categoría. Adicionalmente, la práctica del aikido incluye el desarrollo de otro tipo de destrezas, denominadas ukemi. El ukemi es una forma específica de adaptar el cuerpo a la técnica de control/proyección para disminuir la posibilidad de lesiones. En el programa se incluyó la enseñanza del ukemi específico para la selección de técnicas que se pretendía abarcar. Finalmente, se evitó el uso de toda etiqueta (protocolo de comportamiento) japonesa, como son vestimenta, grados (cintas de colores), reverencias, ambiente solemne, reflexiones y meditación (que son comunes durante la práctica formal de aikido). Solo 12 participantes cumplieron con un mínimo de 10 sesiones al finalizar el programa.

Entre las razones que explican la falta de cumplimiento de todas las sesiones por parte de la mitad de participantes, se destaca la carga académica (por ejemplo, el tener que dedicar tiempo adicional para prepararse para exámenes) y de trabajo (es habitual en esta población estudiantil, el combinar sus responsabilidades académicas con uno o más trabajos que les sirven como fuente de ingreso económico para satisfacer sus necesidades básicas y poder así estudiar). La disminución en la realización de actividades deportivas en estudiantes por falta de tiempo e incompatibilidad por carga académica o laboral, es similar a hallazgos reportados en diversos lugares (Bazán, Martínez, \& Orozco, 2017; Gómez, Ruiz, García, Flores, \& Barbero, 2008; Pérez, 2012; Sanz, Valdemoros, Ramos, \& Ponce de León, 2011).

Mientras tanto, los integrantes del grupo control siguieron con su rutina normal, cursando las materias regulares de su carrera, que en aquel momento incluían tanto cursos teóricos como prácticos, en los que era común la práctica de actividades físicas de moderada a vigorosa intensidad, pero en ningún caso dichas actividades tenían elementos afines a los desarrollados con el grupo experimental (dicho sea de paso, en el plan de estudios del Bachillerato en Ciencias del Movimiento Humano, no se incluían en aquel momento, cursos de artes marciales, ni siquiera como materia optativa, y además, ninguno de los integrantes del grupo control tenía experiencia en artes marciales).

Para que el diseño estadístico quedara equilibrado, se analizaron los datos de las 12 primeras personas control que completaron los instrumentos en el post test. Cuando entró al sistema en la web el doceavo instrumento, se cerraba automáticamente la inclusión de más datos. 


\section{Análisis de datos}

Se calcularon promedios y desviaciones estándar de las variables cuantitativas pertinentes, y previo a los análisis de prueba de hipótesis que más adelante se mencionará, se aplicó la prueba de normalidad Shapiro-Wilk (adecuada para menos de 50 casos, según O’Donoghue, 2012) encontrándose que la distribución de los datos correspondientes a los distintos momentos de medición de la ansiedad (psíquica, somática y global) difería ( $\mathrm{p}<.05)$ de la distribución normal, situación contraria en los puntajes de mindfulness.

Se aplicó el análisis de varianza de 2 vías mixto (grupos y mediciones) para cada variable dependiente: mindfulness, ansiedad psíquica, somática y global (estas tres últimas variables son calculadas con la HAS). Y se aplicó como post hoc para interacción doble significativa (que es el efecto de interacción entre las variables grupos y mediciones, y que se calcula con el análisis de varianza de 2 vías), el análisis de efectos simples. De acuerdo con O’Donoghue (2012, p.232), en los casos en que se viole el supuesto de normalidad de los datos para un análisis de varianza, si la máxima desviación estándar de los distintos niveles a comparar es menor que el doble de la mínima desviación estándar, entonces se puede considerar la prueba suficientemente robusta para lidiar con el problema de normalidad expuesto (resultados de prueba de Shapiro-Wilk). Dicha condición fue satisfecha para la ansiedad psíquica y la global.

Asimismo se probaron los supuestos de esfericidad (prueba de Mauchly, satisfecha por defecto al haber solo dos mediciones en el diseño) y de homogeneidad (prueba de Levene que fue satisfecha en los puntajes de mindfulness, ansiedad psíquica y global). Además, mediante la prueba de rangos ordenados de Spearman (al no asumirse normalidad), no se encontró relación estadísticamente significativa $(p<.05)$ entre la edad de los participantes y los puntajes de cada medición de las variables estudiadas, y tampoco se encontró diferencias entre las edades de ambos grupos ( $t=-0.986 ; p=.343$; sin asumir varianzas iguales a obtenerse prueba de Levene significativa), por tanto, no se requirió aplicar análisis de covarianza con la edad como covariable.

Finalmente, en el caso de la variable ansiedad somática, al no cumplir con los supuestos de normalidad mencionados, se aplicó la prueba estadística no paramétrica $L$ desarrollada por Puri y Sen $(1969,1985)$ y promovida por Thomas, Nelson y Thomas (1999) y Thomas et al. (2015) para su uso en ciencias del deporte y el ejercicio como alternativa cuando no se cumplen los supuestos de normalidad de una prueba paramétrica como el análisis de varianza.

Para profundizar más en los efectos del programa de aikido, y siguiendo las indicaciones de la sexta edición del manual de publicaciones de la APA (2010) respecto a la importancia de usar intervalos de confianza y medidas del tamaño de efecto en el reporte de resultados (aspecto reforzado por críticas al modelo tradicional de prueba de hipótesis nulas basado en el valor límite $p<.05$, según varios autores como Amrhein, Korner-Nievergelt, \& Roth, 2017; Concato \& Hartigan, 2016; Cumming, 2014; Greenland et al., 2016; Jiroutek \& Turner, 2016; Perezgonzalez, 2015; Ziliak, 2016; además de lo planteado en una reciente declaración de la Asociación Americana de Estadística publicada en Wasserstein \& Lazar, 2016), se calculó el porcentaje de cambio (PC) pretest-postest para las variables: mindfulness, los componentes psíquico (CP) y somático (CS) de la ansiedad, así como para su puntaje global. Para ello se usó la siguiente fórmula:

\section{$P C=[(\bar{x}$ Post $-\bar{x}$ Pre $) / \bar{x}$ Pre $] * 100$}

Y en la misma línea, se calculó el tamaño de efecto(TE) de medidas repetidas para cada grupo, mediante la fórmula(Becker, 1988; Thomas, Nelson y Silverman, 2015):

$$
T E=\frac{\bar{x} \text { Post }-\bar{x} \text { Pre }}{\text { DEpre }} \text { DEpre: desviación estándar del pre test. }
$$

El TE cuantifica el impacto del programa de práctica de aikido, sobre las variables dependientes (Quezada, 2007) y se clasifica como pequeño, moderado y alto, según su magnitud (Cohen, 2013). Los TE fueron corregidos para evitar sesgo² siguiendo lo propuesto por Hedges
(1981) y descrito en Borenstein (2009) y Cooper, Hedges y Valentine (2009). Se calculó intervalos de confianza (IC) al $95 \%{ }^{3}$ para cada TE.

Para tener mayor información destinada a explicar los resultados del experimento, se aplicó la correlación parcial, relacionando los porcentajes de cambio de las variables medidas, controlando la correlación entre el par de variables de interés y las otras dos variables (cuya relación puede afectar la magnitud de la correlación de interés, conduciendo a interpretaciones erróneas).

Los análisis fueron realizados con el Paquete Estadístico para las Ciencias Sociales (SPSS, versión 18.0) y Microsoft Excel 2007.

\section{Resultados}

En la tabla 1 se resume la estadística descriptiva, y en la tabla 2 se resumen los análisis de varianza que se aplicaron, complementados con los TE y sus IC al 95\% de confianza.

Tabla 1.

Resumen de estadística descriptiva (promediołdesviación estándar) de las variables principales del estudio Variables Grupo Control Grupo Experimental Total

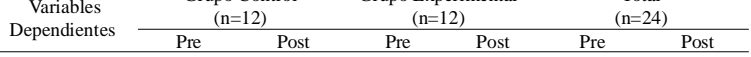

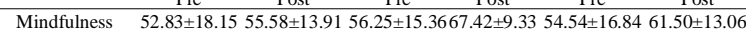
$\begin{array}{lllllll}\text { Ansiedad CPa } & 12.92 \pm 2.71 & 13.00 \pm 3.25 & 14.58 \pm 4.14 & 11.75 \pm 3.96 & 13.75 \pm 3.53 & 12.38 \pm 3.60\end{array}$ $\begin{array}{lllllll}\text { Ansiedad CPa } & 12.92 \pm 2.71 & 13.00 \pm 3.25 & 14.58 \pm 4.14 & 11.75 \pm 3.96 & 13.75 \pm 3.53 & 12.38 \pm 3.60 \\ \text { Ansiedad CS } & 10.00 \pm 2.30 & 11.00 \pm 3.54 & 10.00 \pm 4.65 & 8.00 \pm 1.76 & 10.00 \pm 3.59 & 9.50 \pm 3.13\end{array}$

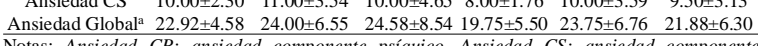
Notas. Ansiedad CP: ansiedad componente psiquico. Ansiedad CS: ansiedad componente

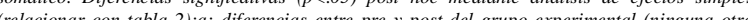
diferencia estadísticamente significativa).

Tabla 2.

Resumen de análisis de varianza de dos vías mixto (grupos v.s. mediciones), aplicados a las Reriables dependientes del estudio, tamaños de efecto (post $v . S$. pre test) e intervalos de confianza

Variables Medición

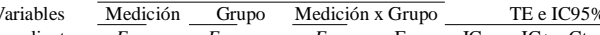

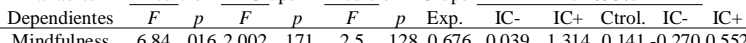

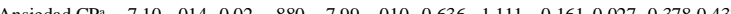

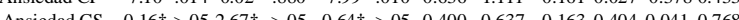

A

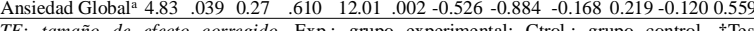
1E. Lamano de efecto corregido. Exp.: grupo expeninental, Ctrol.. grupo control. +Test

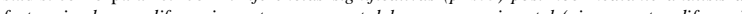
significativa).

Se evidenciaron (tabla 2) efectos positivos de la práctica del aikido sobre mindfulness y el estado de ansiedad. Los IC \pm de los TE al 95\% de confianza del grupo experimental no incluyen al cero, al contrario del grupo control. Por tanto, no hay evidencia de variables extrañas influyentes en los resultados, y estos se pueden atribuir al entrenamiento de aikido.

Pero los resultados de la ansiedad CS deben interpretarse con cautela, pues en este caso se observó TE distinto de cero en el grupo control, indicando la influencia de al menos una variable extraña que aumentó la ansiedad CS en este grupo.

La correlación parcial entre los porcentajes de cambio de las cuatro variables dependientes examinadas, mostró que los cambios en mindfulness estaban poco relacionados con los cambios en ansiedad CP $\left(r_{\text {parcial }}=.61 p=.062\right), \mathrm{CS}\left(r_{\text {parcial }}=.49 p=.147\right)$ y global $\left(r_{\text {parcial }}=-.59\right.$ $p=.070$ ), siendo los cambios en ansiedad CP y global los que más podrían explicar las mejoras en mindfulness en el grupo experimental (varianzas explicadas comunes, tras elevar al cuadrado el estadístico de correlación y multiplicar esto por 100, de 37\% y 35\% respectivamente).

\section{Discusión}

En general, la práctica de aikido mostró efectos significativos estadísticamente y de magnitud moderada tanto en mindfulness como en el puntaje global y en el componente psíquico (CP) de la ansiedad, y efecto significativo y pequeño en el componente somático (CS) de la ansiedad. No obstante, en el caso del mindfulness, la probabilidad de que los datos obtenidos sucedan (es decir, que el resultado observado se presente), siendo verdadera la hipótesis nula de no interacción entre grupos y mediciones (es decir, que el comportamiento de los sujetos experimentales y controles, entre mediciones, sea igual), fue de un $13 \%$ 
( $p=.128$ ), superando el tradicional límite de 5\% aplicado para la decisión de rechazar hipótesis nulas en investigaciones en psicología y otros ámbitos de las ciencias sociales y de la salud. Y aunque no existe realmente una justificación científica, salvo el criterio de autoridades, como las mismas que dieron vida y defendieron al modelo de prueba estadística de hipótesis nulas (Cohen, 1994; Figueiredo et al., 2013), ha sido habitual la aplicación mecánica o ritualista de este modelo, según cuestionan varios autores (Amrhein, Korner-Nievergelt, \& Roth, 2017; Greenland et al., 2016; Kian-Leong, 2009), descartándose los resultados no estadísticamente significativos como no relevantes, pero esta práctica ha sido criticada al punto de que se han llegado a plantear prohibiciones de su uso en ciertas publicaciones científicas del campo de la psicología (Savalei \& Dunn, 2015; Trafimow \& Marks, 2015).

Un mayor desarrollo de este tema, cuyo debate sigue abierto, sobrepasa los alcances del presente trabajo, pero sirva esta breve mención, al menos, para mostrar la importancia de profundizar en la interpretación de los resultados, más allá del ritual de la prueba estadística de hipótesis nula creado por Neyman y Pearson y seguidores, y tratando de recuperar la esencia original de Fisher, creador del modelo de los valores $p$, y quien no pretendió que estos sirvieran para una toma de decisión dicotómica entre aceptar y rechazar hipótesis, y menos aún con un valor fijo como límite, sino que buscaba que los valores $p$ funcionaran como parte de un proceso inferencial que ayudase a los investigadores para medir el apoyo que sus datos dan a su hipótesis en cuestión, facilitándoles pensar qué tan creíble puede ser esta ante aquellos (varios autores respaldan lo anterior e incluso cuestionan críticas que se han lanzado a Fisher, como lo que plantean Greenland et al., 2016; KianLeong, 2009).

Según varios autores (Altman \& Bland, 1995; Sedgwick, 2011), la falta de un resultado estadísticamente significativo no implica la ausencia de un efecto real. De hecho los tamaños de efecto obtenidos para mindfulness y ansiedad evidencian que el programa de aikido puede favorecer esas variables en sujetos sin experiencia previa en artes marciales o ejercicio afín, pero la reducción de la muestra experimental, por razones fuera del manejo de los investigadores, puede ser un aspecto que explique que la interacción entre grupos y mediciones examinada con el análisis de varianza no tuviera un valor $p$ más bajo, dada la influencia que se ha demostrado que existe del tamaño de muestra sobre los valores p (Concato \& Hartigan, 2016; Gelman \& Stern, 2006), de modo que con solo aumentar un poco el $n$ o cambiar de prueba estadística se podría disminuir convenientemente el valor de $p$ (siendo criticables estas prácticas según Amrhein, Korner-Nievergelt, \& Roth, 2017). En síntesis: se debe prestar más atención a la evidencia respecto a la magnitud y variabilidad de los tamaños de efecto encontrados.

Los hallazgos del presente estudio, además, contradicen lo encontrado en investigaciones previas como la de Foster (1997) y Tapley (2007). En el caso del estudio de Foster (1997), se compararon estudiantes de aikido con practicantes de karate y de golf (los tres grupos eran de estudiantes universitarios voluntarios de cursos de educación física para principiantes), y luego de 10 semanas de entrenamiento se encontró que solo los karatecas mejoraron significativamente en la ansiedad rasgo. De hecho solo en esa variable hubo diferencias, pues en las demás (autoestima, ansiedad estado e ira) ningún grupo mejoró. Pero faltó mayor detalle de los procedimientos en este estudio, además de que no queda claro por qué se realizaron múltiples análisis de t-Student para comparar las mediciones pre v.s. post entrenamiento, en cada uno de los grupos, en lugar de haberse realizado el análisis de varianza mixto de dos vías como correspondía para el diseño estadístico de este trabajo.

Por otro lado, en el caso de Tapley (2007), su estudio fue correlacional, operacionalizando la práctica de aikido como el rango de cinturón, siendo esta característica la que no se relacionó con síntomas somáticos, estrés percibido, ansiedad ni espiritualidad (por el contrario se encontró que el rango de cinturón en aikido predecía mayor percepción de dolor en la espalda y otras zonas del cuerpo usualmente sometidas a impactos en esta práctica; el único aspecto positivo que se relacionó con el rango de cinturón fue la firmeza o estabilidad, aspecto valorado con el ítem 19 del Trait Anxiety Inventory de Spielberger, y de hecho el único ítem que se relacionó significativamente con el rango de cinturón). El estudio de Tapley tiene las debilidades inherentes a cualquier estudio correlacional, en cuanto a su incapacidad para concluir relaciones causa-efecto, pero además, el hecho deusar el rango de cinturón como estándar de conocimiento o experiencia en la práctica del aikido (tal y como se ha hecho en otros estudios, como en Lothes et al., 2013) tiene la limitante de que, si bien se puede asumir un número mínimo de horas de práctica para alcanzar cada rango, no es posible controlar la cantidad de práctica real de aikido (ni delimitarla solo como práctica física, pues en el entrenamiento regular se incluyen además prácticas ceremoniales y de meditación que también pueden tener un efecto en procesos afectivos y cognitivos), ni se puede determinar que todos los sujetos de cierto rango hayan realizado la misma cantidad de ejercicio.

Por otro lado, se ha mostrado que los practicantes de aikido y de capoeira, tienen mejor perfil de conductas relacionadas a la salud, en comparación con sujetos no deportistas (Boguszewski, Adamczyk, Suchcicka, Slyk, \& Bialoszewski, 2014), y esto indica que los practicantes de aikido pueden tener estilos de vida más saludables que sujetos no deportistas (lo cual puede ser de hecho, una característica común a cualquier deportista o sujeto habituado al ejercicio físico). Por tanto, es posible que la reducción en la ansiedad encontrada en el presente estudio, se deba a diversos factores inherentes a la práctica del aikido, como sistema de ejercicio físico, y que cambios, incluso pequeños en mindfulness asociados a dicha práctica, pueden explicar también un mejor estado anímico. Pero esto se debe profundizar en futuros estudios experimentales, en los cuales se recomienda proceder tal y como se hizo en el presente trabajo, controlando la influencia de variables extrañas como las prácticas de meditación.

Desde el punto de vista de la enacción, paradigma de las ciencias cognitivas planteado por Varela (Varela, 2005; Varela, Thompson, \& Rosch, 1992), la naturaleza de una técnica de aikido representa un sistema dinámico de gran complejidad, que exige centrarse en el momento presente, del cual emerge un darse cuenta (awareness), que ocurre constantemente, momento a momento, permitiendo al practicante analizar la situación y responder, eficientemente y a su favor, a la compleja relación de variables que conforman el mencionado sistema dinámico. Es por esto que la práctica constante parece fortalecer, en teoría, la capacidad de darse cuenta, volviéndola una habilidad indispensable para el aikidoka. Estos argumentos, encuentran respaldo en los resultados del presente estudio. No obstante, en futuras investigaciones, entre otros aspectos, se recomienda examinar el efecto de aumentar el volumen de práctica (más cantidad de sesiones o mayor frecuencia semanal haciendo el entrenamiento más intensivo), dado que esta podría ser una variable moderadora de los efectos del aikido en mindfulness y ansiedad.

También vale mencionar que evitar la etiqueta (protocolo de comportamiento) y las prácticas de meditación durante la intervención, contribuyó a controlar sus posibles efectos. Al respecto, en estudios previos (Foster, 1997; Lothes et al., 2013; Tapley, 2007), parece que los efectos de los ejercicios de aikido estuvieron mezclados con los de otras prácticas como las mencionadas (etiqueta y meditación). Esto profundiza la duda sobre el conocimiento previo en este campo, y resalta la importancia de los resultados del presente estudio, los cuales requieren examinarse más rigurosamente en estudios posteriores, mejorando el diseño experimental, que en el presente caso fue cuasiexperimental, al no poderse crear ambos grupos de manera aleatoria.

Las afirmaciones de Edinborough (2011) y Lothes et al. (2013) se confirman dado el tamaño de efecto de la práctica de aikido sobre mindfulness encontrado en este estudio, además del efecto observado sobre la ansiedad y de la evidencia de que los cambios en mindfulness se puedan explicar por variaciones en ese estado anímico, principalmente en su componente psíquico o cognitivo, acorde con evidencias previas (Orsillo et al., 2004; Roemer \& Orsillo, 2007). Sin embargo, es necesario que se realicen más estudios para verificar esta asociación, sobre todo considerando la magnitud de los valores $p$ encontrados para las correlaciones parciales entre los porcentajes de cambio de mindfulness y ansiedad. Al respecto, Cohen (1994), entre otros críticos del tradicional 
modelo de prueba estadística de hipótesis nulas, indica que en psicología debería instaurarse una cultura de la replicación y verificación de resultados, en lugar de seguir confiando en el ritual de la prueba de hipótesis con el arbitrario nivel $p<.05$. Así mismo, no se han encontrado antecedentes de investigaciones previas de tipo experimental en que se aplique el entrenamiento en aikido para la afectación de ambos procesos (afectivos y cognitivos) y como se ha podido demostrar, dicha práctica puede afectar positivamente y simultáneamente a ambos. Por lo tanto, se recomienda realizar más estudios para profundizar el conocimiento generado por la presente investigación.

Es importante señalar, que el 100\% de la muestra (tanto el grupo control como el experimental) reportó tener el hábito de realizar actividad física por lo menos 3 horas por semana, y este dato en particular refuerza la validez de la intervención, dado el ya ampliamente investigado efecto regulador que ejerce la actividad física sobre la ansiedad (Azevedo et al., 2012; Ensari et al., 2015; Fox, 1999; Wegner et al., 2014), pero los resultados muestran que el practicar aikido (tal y como podría suceder con otras artes marciales) aporta un beneficio en el estado anímico que supera el que se puede obtener del hábito regular de actividad física, como parte de un estilo de vida activo (como era el caso de los sujetos controles quienes eran estudiantes universitarios de la carrera de educación física). Pero nuevamente se señala la necesidad de profundizar esta línea de estudio.

\section{Agradecimientos}

Estainvestigación fue financiada con unabeca mixta delCONACYT y con apoyo por estancia a investigador participante en el Proyecto 151918 CONACYT - CB 2010

\section{Referencias}

Altman, D.G., \& Bland, J.M. (1995). Absence of evidence is not evidence of absence. BMJ, 311, 485.

American Psychological Association [APA]. (2010). Publication Manual of the American Psychological Association (6 ed.). Washington, DC: Autor.

Araya-Vargas, GA., Gapper-Morrow, S., Moncada-Jiménez, J., \& Buckworth, J. (2009). Translation and Cross-Cultural Validation of the Spanish Version of the Mindful Awareness Attention Scale (MAAS): An Exploratory Analysis and Potential Applications to Exercise Psychology, Sport and Health. International Journal of Applied Sports Sciences, 21(1), 94-114.

Amrhein, V., Korner-Nievergelt, F., \& Roth, T. (2017). The earth is flat ( $>$ > 0.05): significance thresholds and the crisis of unreplicable research. PeerJ, 5, e3544. doi:10.7717/peerj.3544

Asociación Catalana para el Tratamiento de la Ansiedad y Depresión [ACTAD] (s.f.). Escala de ansiedad de Hamilton. [publicación en línea]. Disponible en: http://www.actad.org/actad_cas/test_cas/ test_hamilton.php

Azevedo Da Silva, M., Singh-Manoux, A., Brunner, E. J., Kaffashian, S., Shipley, M. J., Kivimäki, M., \& Nabi, H. (2012). Bidirectional association between physical activity and symptoms of anxiety and depression: the Whitehall II study. European Journal of Epidemiology, 27(7), 537-546. doi:10.1007/s10654-012-9692-8

Bazán, A., Martínez, I., \& Orozco, B. (2017). Participación en actividades físico-deportivas, motivos, demandas e intereses, en estudiantes de Psicología. Revista de Investigación Apuntes Psicológicos, 2(1). Disponible en: http://revistascientificas.upeu.edu.pe/ index.php/ri_apsicologia/article/view/670

Baer, R.A. (2003). Mindfulness training as a clinical intervention: A conceptual and empirical review. Clinical Psychology: Science and Practice, 10(2), 125-143. doi: 10.1093/clipsy.bpg015

Barajas, S. \& Garra, L. (2014). Mindfulness and psychopathology: Adaptation of the Mindful Attention Awareness Scale (MAAS) in a Spanish sample. Clínica y Salud, 25(2014), 49-56. doi: http:// dx.doi.org/10.5093/cl2014a4
Becker, B.J.(1988). Synthesizing standardized mean-change measures. British Journal of Mathematical and StatisticaI Psychology, 41, 257-278.

Bodner, T. E., \& Langer, E. J. (2001, Junio). Individual differences in mindfulness: The Mindfulness/Mindlessness Scale. Poster presentado enla

13 convención anual de la American Psychological Society, Toronto, Ontario, Canada.

Boguszewski, D., Adamczyk, J.G., Suchcicka, B., Syk, E., \& Biaoszewski,D. (2014). The estimation of health-related behaviours of men practising aikido and capoeira. «IDO MOVEMENT FOR CULTURE. Journal of Martial Arts Anthropology», 14(2), 41-46. doi: 10.14589/ido.14.2.6

Borenstein, M. (2009). Effect sizes for continuous data. En H. Cooper, L.V. Hedges, \& J.C. Valentine [Eds.], The handbook of research synthesis and meta-analysis (2 ed.). New York: Russell Sage Foundation.

Brown, K. W. \& Ryan, R. M. (2003). The benefits of being present: mindfulness and its role in psychological well-being. Journal of Personality and Social Psychology, 84(4), 822-848. doi:10.1037/ 0022-3514.84.4.822

Carlson, L.E. \& Garland, S.N. (2005). Impact of mindfulness-based stress reduction (MBSR) on sleep, mood, stress and fatigue symptoms in cancer outpatients. International Journal of Behavioral Medicine, 12(4), 278-285. doi:10.1207/ s15327558ijbm1204 9

Carrobles I., J.A.; Costa C., M.; Del Ser, T., \& Bartolomé, P. (1987). La práctica de la terapia de conducta: teoría y método de aplicación para la práctica clínica (1 ed., 7 reimp.). Valencia: Promolibro.

Cebolla, A., \& Miró, M.T. (2007). Eficacia de la Terapia Cognitiva basada en la Atención Plena en el tratamiento de la depresión. Revista de Psicoterapia, 17(66/67), 133-155.

Cohen, J. (1994). The Earth is round $(p<.05)$. American Psychologist, 49(12), 997-1003.

Cohen, J. (2013). Statistical Power Analysis for the Behavioral Sciences (ed. rev.). New York: Academic Press.

Concato, J. \& Hartigan, J.A. (2016). P values: from suggestion to superstition. J. Investig. Med., 64,1166-1171. doi:10.1136/jim2016-000206

Cooper, H., Hedges, L.V. \& Valentine, J.C. [Eds.] (2009). The handbook of research synthesis and meta-analysis (2 ed.). New York: Russell Sage Foundation.

Cumming, G. (2014). The new statistics: why and how. Psychological Science, 25(1) 7-29. doi: 10.1177/09567976135049

Davidson, R.J., Kabat-Zinn, J., Schumacher, J., Rosenkranz, M., Muller, D., Santorelli, S.F., .. Sheridan, J.F. (2003). Alterations in brain and immune function produced by mindfulness meditation. Psychosomatic Medicine, 65(4), 564-570. doi:10.1097/ 01.PSY.0000077505.67574.E3

De la Fuente A., J.; Franco J., C., \& Mañas M., I. (2010). Efectos de un programa de entrenamiento en conciencia plena (mindfulness) en el estado emocional de estudiantes universitarios. Estudios sobre Educación, 2010(19), 31-52.

Delgado P., L.C. (2009). Correlatos psicofisiológicos de mindfulness y la preocupación. Eficacia de un entrenamiento en habilidades mindfulness. (Tesis Doctoral publicada). Universidad de Granada, Granada: Editorial de la Universidad de Granada. Disponible en: http://digibug.ugr.es/bitstream/10481/2177/1/17855044.pdf

Dobson, T. \& Miller, V. (1993). Aikido in Everyday Life. Giving in to Get Your Way (Rep Sub edition). Berkeley, CA: North Atlantic Books.

Edinborough, C. (2011). Developing decision-making skills for performance through the practice of mindfulness in somatic training. Theatre, Dance and Performance Training, 2(1), 18-33. doi:10.1080/19443927.2010.543917

Ensari, I., Greenlee, T.A., Motl, R.W., \& Petruzzello, S.J.(2015). Metaanalysis of acute exercise effects on state anxiety: an update of 
randomized controlled trials over the past 25 years. Depression and Anxiety, 32(8), 624-634. doi: 10.1002/da.22370

Figueiredo F., D.B.; Paranhos , R.; da Rocha, E.C.; Batista , M.; da Silva Jr., J.A.; Wanderley D.S. , M.L., \& Guiro M., J. (2013). When is statistical significance not significant? Brazilian Political Science Review, 7(1), 31 - 55.

Foster, Y.A. (1997). Brief aikido training versus karate and golf training and university students' scores on self-esteem, anxiety, and expression of anger. Perceptual and Motor Skills, 84, 609-610.

Fox, K. R. (1999). The influence of physical activity on mental wellbeing. Public health nutrition, 2(3a), 411-418.

Gelman, A. \& Stern, H. (2006). The difference between «significant» and «not significant» is not itself statistically significant. The American Statistician, 60(4), 328-331. doi: 10.1198/ 000313006X152649

Greenland, S., Senn, S.J., Rothman, K.J., Carlin, J.B., Poole, C., Goodman, S.N., \& Altman, D.G (2016). Statistical tests, P values, confidence intervals, and power: a guide to misinterpretations. Eur J Epidemiol, 31, 337-350. doi:10.1007/s10654-016-0149-3

González C., G., Valdivia M., P.A., Cachón Z., J., Zurita O., F., \& Romero R., O. (2017). Influencia del control del estrés en el rendimiento deportivo: la autoconfianza, la ansiedad y la concentración en deportistas. RETOS. Nuevas Tendencias en Educación Física, Deporte y Recreación, 32, 3-6.

Gómez, M., Ruiz, F., García, M., Flores, G, \& Barbero, G. (2008). Razones que influyen en la inactividad físico-deportiva en la Educación Secundaria Post Obligatoria. Retos. Nuevas Tendencias en Educación Física, Deporte y Recreación, 14, 80-85.

Hamilton, M. (1959). The assessment of anxiety states by rating. British Journal of Medicine and Psychology, 32(1), 50-55.

Hedges, L.V. (1981). Distribution theory for Glass's estimator of effect size and related estimators. Journal of Educational Statistics, 6(2), 107-128.

Hernández S., R., Fernández C., C., \& Baptista L., P. (2014). Metodología de la investigación (6 ed.). México, D.F.: McGraw-Hill.

Jiroutek, M.R. \& Turner, J.R. (2016). In praise of confidence intervals: Much more informative than p values alone. The Journal of Clinical Hypertension, 18(9), 955-957. doi: 10.1111/jch.12908

Kian-Leong, C. (2009). The cult of statistical significance: how the standard error costs us jobs, justice, and lives. Journal of Economic Methodology, 16(4), 431-434, doi:10.1080/13501780902943566

Franco J., C. \& Navas L., M. (2009). Efectos de un programa de meditación en los valores de una muestra de estudiantes universitarios. Electronic Journal of Research in Educational Psychology, 7(19), 1157-1174.

Kabat-Zinn, J. (1982). An outpatient program in behavioral medicine for chronic pain patients based on the practice of mindfulness meditation: Theoretical considerations and preliminary results. General Hospital Psychiatry, 4(1), 33-47.

Kabat-Zinn, J. (1990). Full catastrophe living: The program of the stress reduction clinic at the University of Massachusetts Medical Center. New York: Delta.

Kabat-Zinn, J. (2003). Mindfulness-based interventions in context: past, present, and future. Clinical Psychology: Science and Practice, 10(2), 144-156.

Kabat-Zinn, J. (2005). Vivir con plenitud las crisis. Cómo utilizar la sabiduría del cuerpo y de la mente para afrontar el estrés, el dolor y la enfermedad. Barcelona: Editorial Kairós.

Kumar, S., Feldman, G, \& Hayes, A. (2008). Changes in mindfulness and emotion regulation in an exposure-based cognitive therapy for depression. Cognitive Therapy and Research, 32(6), 734-744. doi: 10.1007/s10608-008-9190-1

Looney, M.A., Feltz, C.J., \& VanVleet, C.N. (1994). The Reporting and Analysis of Research Findings for Within-Subject Designs: Methodological Issues for Meta-Analysis. Research Quarterly for Exercise and Sport, 65(4), 363-366. doi: 10.1080/ 02701367.1994.10607641
López-Maya, E.; Hernández-Pozo, M.R.; Méndez-Segundo, L.; Gutiérrez-García, J.J.; Araujo-Díaz, D.; Núñez-Gazcón, A.,...Hölzel, B.K. (2015). Psychometric properties of the mexican version of the mindful attention awareness scale (MAAS). Psychol. Av. Discip., 9(1, Enero-Junio), 13-22.

Lothes II, J., Hakan, R., \& Kassab, K. (2013). Aikido experience and its relation to mindfulness: a two-part study. Perceptual and Motor Skills, 116(1), 30-39. doi:10.2466/22.23.PMS.116.1.30-39

Lutz, J., Brühl, A.B., Scheerer, H., Jäncke, L., \& Herwig, U. (2016). Neural correlates of mindful self-awareness in mindfulness meditators and meditation-naïve subjects revisited . Biological Psychology, 119 (2016), 21-30. doi: http://dx.doi.org/10.1016/ j.biopsycho.2016.06.010

Majumdar, M., Grossman, P., Dietz-Waschkowski, B., Kersig, S., \& Walach, H. (2002). Does mindfulness meditation contribute to health? Outcome evaluation of a German sample. The Journal of Alternative \& Complementary Medicine, 8(6), 719-730.

Malmgren-Olsson, E.B., Armelius, B.A., \& Armelius, K. (2001). A comparative outcome study of body awareness therapy, Feldenkrais, and conventional physiotherapy for patients with nonspecific musculoskeletal disorders: Changes in psychological symptoms, pain, and self-image. Physiotherapy Theory and Practice, 17(2), 77-95.

Marlatt, G.A. \& Kristeller, J.L.. (1999). Mindfulness and meditation. En W. R. Miller (Ed.). Integrating spirituality into treatment: Resources for practitioners, (pp. 67-84). Washington, D.C.: American Psychological Association.

National Institutes of Health (octubre, 2010). Fact Sheet. Mind-Body Medicine Practices in Complementary and Alternative Medicine. [publicación en línea]. Disponible en: https://report.nih.gov/ NIHfactsheets/ViewFactSheet.aspx?csid=102

O’Donoghue, P. (2012). Statistics for sport and exercise studies. An introduction. New York: Routledge.

Orsillo, S. M., Roemer, L., Block-Lerner, J., \& Tull, M. T. (2004). Acceptance, mindfulness, and cognitive-behavioral therapy: Comparisons, contrasts, and application to anxiety. En Steven C. Hayes, Victoria M. Follette, \& Marsha M. Linehan (Eds), Mindfulness and Acceptance: Expanding the Cognitive-Behavioral Tradition, (pp. 66-95). New York: Guilford. New York: Guilford Press.

Perezgonzalez, J.D. (2015). Fisher, Neyman-Pearson or NHST? A tutorial for teaching data testing. Frontiers in Psychology, 6(artículo 223), 1-11. doi: 10.3389/fpsyg.2015.00223

Pérez, J. (2012). Motivaciones y etapas de cambio de comportamiento ante la actividad físico-deportiva en estudiantes de la Universidad Autónoma de Nuevo León, México. Tesis Doctoral, Universidad Pablo de Olavide de Sevilla, España.

Pérez-Ordás, R., Hernández-Hernández, E., \& García-Sánchez, I. (2011). Relación entre el nivel de habilidad motriz y la ansiedad percibida antes y después de una práctica de escalada en niños de Educación Primaria. RETOS. Nuevas Tendencias en Educación Física, Deporte y Recreación, 19, 25-29.

Petruzzello, S.J., Landers, D.M., Hatfield, B.D., Kubitz, K.A., \& Salazar, W.(1991). Ameta-analysis on the anxiety-reducing effects of acute and chronic exercise. Outcomes and mechanisms. Sports Med., 11(3), 143-82.

Pieter, W. \& Pieter, M.S. (2008). Mood and performance in aikido athletes. Acta Kinesiologiae Universitatis Tartuensis, 13, 107-116. doi: http://dx.doi.org/10.12697/akut.2008.13.09

Puri, M.L. \& Sen, P.K. (1969). A class of rank order tests for a general linear hypothesis. Annals of Mathematical Statistics, 40, 13251343.

Puri, M.L. \& Sen, P.K. (1985). Nonparametric methods in general linear models. New York: Wiley.

Quezada, C. (2007). Potencia estadística, sensibilidad y tamaño de efecto: ¿un nuevo canon para la investigación?, Onomázein, 2(16), 159-170. 
Rodriguez-Pomeda, J., Casani, F., \& Sandoval Hamón, L. (2018). Emotions and the sustainability of community sport organizations. Journal of Human Sport and Exercise, 13(1), 149-160. doi: https:/ /doi.org/10.14198/jhse.2018.131.15

Roemer, L. \& Orsillo, S. M. (2007). An open trial of an acceptancebased behavior therapy for generalized anxiety disorder. Behavior Therapy, 38(1), 72-85.

Röthlin P., Birrer D., Horvath S., \& Grosse Holtforth M. (2016). Psychological skills training and a mindfulness-based intervention to enhance functional athletic performance: design of a randomized controlled trial using ambulatory assessment. B.M.C. Psychol., 4(1, Julio), 39. doi: 10.1186/s40359-016-0147-y.

Salmon, P., Lush, E., Jablonski, M., \& Sephton, S.E. (2009). Yoga and mindfulness: Clinical aspects of an ancient mind/body practice. Cognitive and Behavioral Practice, 16(1), 59-72.

Saotome, M. (1986/2013). Aikido and the harmony of nature. Boston y Londres: Shambhala.

Saotome, M. (1989). The principles of aikido. Boston y Shaftesbury: Shambhala.

Savalei, V., \& Dunn, E. (2015). Is the call to abandon p-values the red herring of the replicability crisis? Front. Psychol, 6,245. doi: 10.3389/ fpsyg.2015.00245

Sedgwick, P. (2011). Absence of evidence is not evidence of absence. BMJ, 342, d3126. doi: 10.1136/bmj.d3126

Sanz, E., Valdemoros, M., Ramos, R., \& Ponce de León, A. (2011) Claves educativas para aumentar la motivación de los jóvenes físicamente inactivos. European Journal of education and psychology. 4(2), 109-118.

Segal, Z.V.; Williams, J.M.G., \& Teasdale, J.D. (2006). Terapia cognitiva de la depresión basada en la consciencia plena. Un nuevo abordaje para la prevención de las recaídas. Bilbao: Desclée de Brouwer.

Shapiro, S.L., Astin, J.A., Bishop, S.R., \& Cordova, M. (2005). Mindfulness-based stress reduction for health care professionals: results from a randomized trial. International Journal of Stress Management, 12(2), 164-176.

Shapiro, S.L., Carlson, L.E., Astin, J.A., \& Freedman, B. (2006) Mechanisms of mindfulness. Journal of Clinical Psychology, 62(3), 373-386. doi: 10.1002/jclp.20237

Sharon, H., Maron-Katz, A., Ben Simon, E., Flusser, Y., Hendler, T., Tarrasch, R., \& Brill, S. (2016). Mindfulness Meditation Modulates Pain Through Endogenous Opioids. Am. J. Med., 129(7, Julio), 755-8. doi: 10.1016/j.amjmed.2016.03.002. Epub 2016 Apr 1.

Shioda, G. (1968) Dynamic aikido. Tokyo: Kodansha International.

Shioda, G. (1991). Aikido Shugyo. Harmony in confrontation. Toronto: Shindokan International.

Soler, J., Tejedor, R., Feliu-Soler, A., Pascual, J.C., Cebolla, A., Soriano, J.,...Pérez, V. (2012). Psychometric properties of Spanish version of Mindful AttentionAwareness Scale(MAAS). Actas Esp Psiquiatr, 40(1), 19-26.

Tang, Y.Y., Hölzel, B.K., \& Posner, M.I. (2015). The neuroscience of mindfulness meditation. Nature Reviews Neuroscience, 16(2015, abril), 213-225. doi:10.1038/nrn3916

Tapley, H.E. (2007). A study of perceived stress, anxiety, somatic symptoms, and spirituality in practitioners of the martial art aikido. (Tesis de doctorado). University of Alabama en Birmingham.

Thomas, J.R., Nelson, J.K. \& Thomas, K.T. (1999). A generalized rank-order method fornon-parametric analysis of data from exercise science: a tutorial. Research Quarterly for Exercise and Sport, 70, 11-23.

Thomas, J.R., Nelson, J.K., \& Silverman, S.J. (2015). Researchmethods in physical activity (7 ed.). Champaign, IL: Human Kinetics.

Trafimow, D., \& Marks, M. (2015). Editorial. Basic Appl Soc Psychol, $37,1-2$.

Ueshiba, K. (1988). The spirit of aikido. Tokyo y New York: Kodansha International.

Vallejo P., M.A. (2006). Mindfulness. Papeles del Psicólogo, 27(2), 92-99.
Varela, F.J.(2005). Conocer. Las ciencias cognitivas: tendenciasy perspectivas. Cartografía de las ideas actuales (4 reimp.). Barcelona: Gedisa.

Varela, F.J., Thompson, E., \& Rosch, E. (1992). De cuerpo presente: las ciencias cognitivas y la experiencia humana. Gedisa.

Volanen, S.M., Lassander, M., Hankonen, N., Santalahti, P., Hintsanen, M., Simonsen, N., ... Suominen, S. (2016). Healthy Learning Mind - a school-based mindfulness and relaxation program: a study protocol for a cluster randomized controlled trial. B.M.C., Psychol., 4(1, Julio), 35. doi: 10.1186/s40359-016-0142-3.

Vøllestad, J.(2016). Mindfulness- and Acceptance-Based Interventions in the Treatment of Anxiety Disorders. En E. Shonin et al. (Eds.), Mindfulness and buddhist-derived approaches in mental health and addiction, Advances in mental health and addiction. Suiza: Springer International Publishing. doi 10.1007/978-3-319-222554_6

Wasserstein, R.L., \& Lazar, N.A. (2016) The ASA's Statement on pvalues: Context, process, and purpose. The American Statistician, 70(2), 129-133. doi:10.1080/00031305.2016.1154108

Wegner, M., Helmich, I., Machado, S., Nardi, A.E., Arias-Carrion, O., \& Budde, H. (2014). Effects of exercise on anxiety and depression disorders: review of meta-analyses and neurobiological mechanisms. CNS \& Neurol Disord - Drug Targets, 13(6), 1002-1014.

Williams, K.A., Kolar, M.M., Reger, B.E., \& Pearson, J.C. (2001). Evaluation of a wellness-based mindfulness stress reduction intervention: A controlled trial. American Journal of Health Promotion, 15(6, Julio/Agosto), 422-432. doi: http://dx.doi.org/ 10.4278/0890-1171-15.6.422

Wipfli, B.M., Rethorst, C.D., \& Landers, D.M. (2008). The anxiolytic effects of exercise: a meta-analysis of randomized trials and doseresponse analysis. J Sport Exerc Psychol., 30(4), 392-410.

Zeidan, F., Adler-Neal, A.L., Wells, R.E., Stagnaro, E., May, L.M., Eisenach, J.C.,...Coghill, R.C. (2016). Mindfulness-meditationbased pain relief is not mediated by endogenous opioids. J.Neurosci., 36(11, Marzo), 3391-7. doi: 10.1523/JNEUROSCI.4328-15.2016.

Zeidan, F., Emerson, N.M., Farris, S.R., Ray, J.N., Jung, Y., McHaffie, J.G., \& Coghill, R.C. (2015). Mindfulness meditation-based pain relief employs different neural mechanisms than placebo and sham mindfulness meditation-induced analgesia. J. Neurosci., 35(46, Noviembre), 15307-25. doi: 10.1523/JNEUROSCI.2542-15.2015.

Zeidan, F. \& Vago, D.R. (2016). Mindfulness meditation-based pain relief: a mechanistic account. Ann. N.Y. Acad. Sci., 1373(1, Junio), 114-27. doi: 10.1111/nyas.13153.

Ziliak, S.T. (2016) Statistical significance and scientific misconduct: improving the style of the published research paper. Review of Social Economy, 74(1), 83-97. doi:10.1080/ 00346764.2016.1150730

\section{(Footnotes)}

1 Se aplicaron versiones en computadora, desarrolladas con tecnologías web: php, mongodb, javascript, css, html5.

2 Se tiende a sobreestimar el TE en muestras pequeñas. Eso se corrige multiplicando el TE por el factor de corrección $J(g l)=1$-[3/(4*gl1)] donde $g l=n-1$ para TE pre v.s. post, y n=tamaño de muestra del grupo (Hedges, 1981; Borenstein, 2009).

3 Previo a esto, se calculó la varianza de cada TE y a partir de esta se calculó el error estándar; vale mencionar que para obtener la varianza, se utilizó en la fórmula la correlación entre las mediciones pre y post, siguiendo lo indicado por Borenstein (2009).

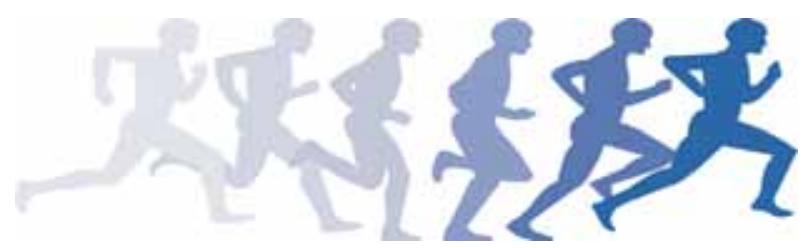

